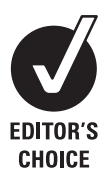

CHOICE

\title{
Visceral fat accumulation is an independent risk factor for hepatocellular carcinoma recurrence after curative treatment in patients with suspected NASH
}

\author{
T Ohki, R Tateishi, S Shiina, E Goto, T Sato, H Nakagawa, R Masuzaki, T Goto, \\ K Hamamura, F Kanai, H Yoshida, T Kawabe, M Omata
}

Department of Gastroenterology University of Tokyo, Tokyo, Japan

Correspondence to: Dr H Yoshida, Department of Gastroenterology, University of Tokyo, 7-3-1 Hongo, Bunkyo-ku, Tokyo 113-8655, Japan; yoshida-2im@h.u-tokyo.ac.jp

Revised 8 January 2009 Accepted 8 January 2009 Published Online First 26 January 2009

\section{ABSTRACT}

Background and Aim: Visceral fat accumulation reportedly increases the risk of hepatocellular carcinoma (HCC) development in patients with chronic liver disease. However, it has not beeen fully elucidated whether visceral fat accumulation increases the risk of HCC recurrence after curative treatment in patients with suspected non-alcoholic steatohepatitis (NASH). Therefore this was investigated in the current study. Methods: 62 patients with naive HCC with suspected NASH were enrolled. All were curatively treated with percutaneous radiofrequency ablation between 1999 and 2006. The visceral fat area (VFA) was determined in each patient from CT images, taken at the time of HCC diagnosis. Patients were divided into two groups based on VFA: the high VFA group $\left(>130 \mathrm{~cm}^{2}\right.$ in males, $>90 \mathrm{~cm}^{2}$ in females, $n=27)$ and the others $(n=35)$. The effects of VFA on HCC recurrence were analysed together with other factors including patients' background, tumourrelated factors and liver function-related factors.

Results: The cumulative recurrence rates differed significantly between the two groups; 15.9, 56.5 and $75.1 \%$ at 1,2 and 3 years, respectively, in the high VFA group, and $9.7,31.1$ and $43.1 \%$, respectively, in the controls $(p=0.018)$. Multivariate analysis indicated visceral fat accumulation (risk ratio 1.08 , per $10 \mathrm{~cm}^{2}$, $p=0.046$ ) and older age (risk ratio 1.06 per 1 year, $p=0.04$ ) as independent risk factors of HCC recurrence. Conclusions: Visceral fat accumulation is an independent risk factor of HCC recurrence after curative treatment in patients with suspected NASH.

Hepatocellular carcinoma (HCC) is the fifth leading cause of cancer death worldwide, accounting for $>500000$ deaths annually, showing an increasing incidence throughout the world. ${ }^{2}{ }^{2}$ HCC usually develops in patients with advanced liver fibrosis due to chronic liver diseases such as chronic hepatitis B, chronic hepatitis C, alcoholic liver disease $^{23}$ and haemochromatosis. ${ }^{4}$ In addition, non-alcoholic steatohepatitis (NASH) is considered to be another important liver disease preceding cirrhosis and HCC. Non-alcoholic fatty liver disease (NAFLD), increasing in prevalence in Western countries as well as in Japan because of the increasing prevalence of obesity, ${ }^{5}$ is shown to be a clinical condition that may progress to NASH and, subsequently, HCC. ${ }^{\circ}$

Recently, obesity has also been reported to be a risk factor of HCC development in patients with chronic liver diseases other than NASH. ${ }^{7-9}$ Emerging data have indicated that neuroendocrine systems that regulate weight and energy metabolism play a pivotal role in the development of hepatic steatosis. ${ }^{10}$ Several mechanisms have been proposed by which accumulation and anatomic distribution of fat are related to the development of hepatic steatosis and fibrosis. ${ }^{11}$ Eguchi et al reported that the severity of fatty liver was positively related to visceral fat accumulation and insulin resistance in both obese and non-obese NAFLD patients without viral hepatitis. ${ }^{11}$ This suggests that hepatic fat infiltration in NAFLD may be influenced by visceral fat accumulation independently of body mass index (BMI).

Based on these findings, we hypothesised that the previously reported relationship between BMI and the risk of HCC was mediated by visceral fat accumulation, and the latter is directly associated with HCC development. Considering the low incidence of HCC among patients with NAFLD in general, the study will require a very large cohort, where it would be difficult to measure visceral fat accurately in each subject. On the other hand, abdominal CT, with which visceral fat accumulation can be calculated, is performed routinely on HCC patients. Since recurrence of HCC is very frequent, the effect of visceral fat can be evaluated in a relatively small cohort. Thus, we conducted this study enrolling consecutive, nonviral non-alcoholic patients with naïve HCC who received curative radiofrequency ablation (RFA) treatment to evaluate the impact of visceral fat accumulation on HCC recurrence.

\section{PATIENTS AND METHODS \\ Patients}

A total of 777 patients received RFA as the treatment for naïve HCC at the authors' institution between January 1999 and December 2006. There were 596 patients positive for hepatitis C virus HCV antibody (HCVAb), 74 patients positive for hepatitis B surface antigen ( $\mathrm{HBs} A g$ ) and 6 patients positive for both HCVAb and HBsAg. A past history of alcohol consumption of $>20 \mathrm{~g}$ ethanol per day was found in another 27 patients, and intractable ascites, which may interfere with visceral fat measurement, was found in 12 on CT scan at diagnosis. Excluding these patients, we enrolled the remaining 62 patients as a cohort and analysed the relationship between visceral fat accumulation and intrahepatic recurrence of HCC. All of these patients were negative for antimitochondrial antibody and antinuclear antibody. Haemochromatosis was not diagnosed in 
any of them. Diagnosis of diabetes mellitus was based on medical history or a $75 \mathrm{~g}$ oral glucose tolerance test. Dyslipidaemia was defined as blood total cholesterol concentration $>220 \mathrm{mg} / \mathrm{dl}$ or triglyceride $>150 \mathrm{mg} / \mathrm{dl}$, or a history of taking oral drugs for dyslipidaemia. BMI was calculated as body weight in kilograms $(\mathrm{kg})$ divided twice by body height in metres (m), which was also routinely measured at the first visit of each patient.

\section{Diagnosis of HCC}

Contrast-enhanced dynamic CT was performed on patients with suspected HCC detected by ultrasonography. Multidetector row CT with 4, 8 or 16 detector rows (Aquilion 4/16; Toshiba, Tokyo, Japan; LightSpeed Ox/I, LightSpeed Ultra; GE Healthcare, Milwaukee, Wisconsin, USA) was used. Images were obtained in early arterial, late arterial and equilibrium phases at 28, 40 and $120 \mathrm{~s}$ after starting bolus injection of iodinated contrast material. Images were reconstructed with a section thickness of $5 \mathrm{~mm}$ and a reconstruction interval of $5 \mathrm{~mm}$ (a section thickness of 2-2.5 mm, an interval of 1.5-2 mm and a field of view (FOV) of $24-35 \mathrm{~cm}$ for arterial phases). The diagnosis of HCC was based on typical findings on CT-that is, hyperattenuation in the arterial phase and hypoattenuation in the equilibrium phase. $^{12}{ }^{13}$ Ultrasoundguided biopsy of the non-tumorous liver was performed in most cases, except for patients with a risk of haemorrhage such as severe thrombocytopenia. Background tissue was pathologically graded based on NAFLD activity score ${ }^{14}$ and Ishak fibrosis staging. ${ }^{15}$ Ultrasound-guided tumour biopsy was also applied when CT findings were inconclusive regarding HCC. HCC tissue was pathologically graded based on Edmondson-Steiner criteria. $^{16}$

\section{Measurement of visceral fat, subcutaneous fat and waist circumference}

Fat tissue area and waist circumference were measured in each patient by analysing a CT image at the level of the umbilicus with the software Slim Vision (KGT, Tokyo, Japan). ${ }^{17}$ The subcutaneous fat area (SFA) was defined as the sum of extraperitoneal fat area between the skin and muscle on the CT image, which showed attenuation ranging from -150 to -50 Housefield units. The visceral fat area (VFA) was defined as the sum of the intraperitoneal fat area showing the same attenuation. The patients were divided into two groups according to the cut-off levels of VFA recommended by Oka et al for metabolic syndrome in the Japanese population ${ }^{18}$ : the high VFA group (defined as VFA $>130 \mathrm{~cm}^{2}$ in males and VFA $>90 \mathrm{~cm}^{2}$ in females, $\left.\mathrm{n}=27\right)$ and the controls $(\mathrm{n}=35)$.

\section{Treatment and follow-up}

The indication criteria for RFA consisted of total bilirubin concentration $<3.0 \mathrm{mg} / \mathrm{dl}$, platelet count $>50 \times 10^{3} / \mathrm{mm}^{3}$ and prothrombin activity $>50 \%$. Patients with portal vein tumour thrombosis, massive refractory ascites or extrahepatic metastasis were excluded. In general, RFA was performed on patients with three or fewer lesions, each $<3.0 \mathrm{~cm}$ in diameter. However, we also performed ablation on patients who did not meet these criteria when complete ablation could be anticipated in all tumours without deteriorating liver function. The detailed procedure was meticulously described elsewhere. ${ }^{19}$ In brief, percutaneous RFA was performed using internally cooled tip RF electrodes with a 2 or $3 \mathrm{~cm}$ long exposed metallic tip (Radionics, Burlington, Massachusetts, USA) on patients after intravenous administration of pentazocine (30 mg), hydroxyzine (25 mg) and atropine $(0.5 \mathrm{mg})$. After local anaesthesia, the electrode was inserted under ultrasound guidance. During ablation, the tip temperature was monitored and kept below $20^{\circ} \mathrm{C}$ with cold saline. In the case of $3 \mathrm{~cm}$ tip electrodes, the output started at $60 \mathrm{~W}$, was increased at $20 \mathrm{~W} / \mathrm{min}$ until tissue impedance overshot and then was decreased by $20 \mathrm{~W}$ and maintained for $12 \mathrm{~min}$. With $2 \mathrm{~cm}$ tip electrodes, the output started at $40 \mathrm{~W}$, was increased at $20 \mathrm{~W} / \mathrm{min}$ until impedance overshot and then was maintained for $6 \mathrm{~min}$.

After RFA, dynamic CT was performed with a section thickness of $0.5 \mathrm{~cm}$ to evaluate treatment efficacy. Complete ablation was defined as hypoattenuation of the whole lesion together with the surrounding liver parenchyma as a safety margin. Patients received additional RFA until complete ablation was confirmed for each HCC nodule.

Major complications were defined as those which, if left untreated, might threaten the patient's life, lead to substantial morbidity and disability, or result in hospital admission or substantially lengthen hospital stay according to the previously described guideline. ${ }^{20-22}$

The follow-up consisted of monthly blood tests and monitoring of tumour markers (alpha-fetoprotein (AFP), lens culinaris agglutinin-reactive fraction of AFP (AFP-L3), and desgamma-carboxy prothrombin (DCP)) at the outpatient clinic, with ultrasonography and dynamic CT scan performed every 4 months. Tumour recurrence was diagnosed based on the same criteria applied to the naïve HCC. Intrahepatic HCC recurrence was classified as recurrence either at a site distant from the primary tumour or adjacent to the treated site (local tumour progression).

\section{Analysis of recurrence}

The end points consisted of the interval between the first ablation and the detection of HCC recurrence, death without recurrence or the last examination until 31 December 2007, whichever came first. Death without recurrence was treated as censored data. Cumulative recurrence of HCC was estimated using the Kaplan-Meier method and compared with log-rank test. The effect of VFA on recurrence was assessed with a multivariate Cox proportional hazard regression model controlling for factors shown to be significant in univariate analysis. ${ }^{23}$ We compared the number and size of tumours at the time of recurrence between the high VFA group and the controls. Changes in VFA between the time of first diagnosis of HCC and at recurrence were also compared in patients with HCC recurrence.

\section{Analysis of survival}

Survival time was defined as the interval between the first treatment and death or the last visit to the outpatient clinic up to 31 December 2007. A cumulative survival curve was plotted by the Kaplan-Meier method, and the difference between the groups was assessed by log-rank test. The effect of VFA on survival was assessed by multivariate Cox proportional hazard regression adjusted for factors shown to be significant in univariate analysis.

\section{Statistical analysis}

We used the following variables obtained at the initial RFA in the analysis of recurrence and survival: age, gender, tumour factors including size and number of nodules, BMI, VFA, SFA, waist circumference, aspartate aminotransferase (AST) level, 

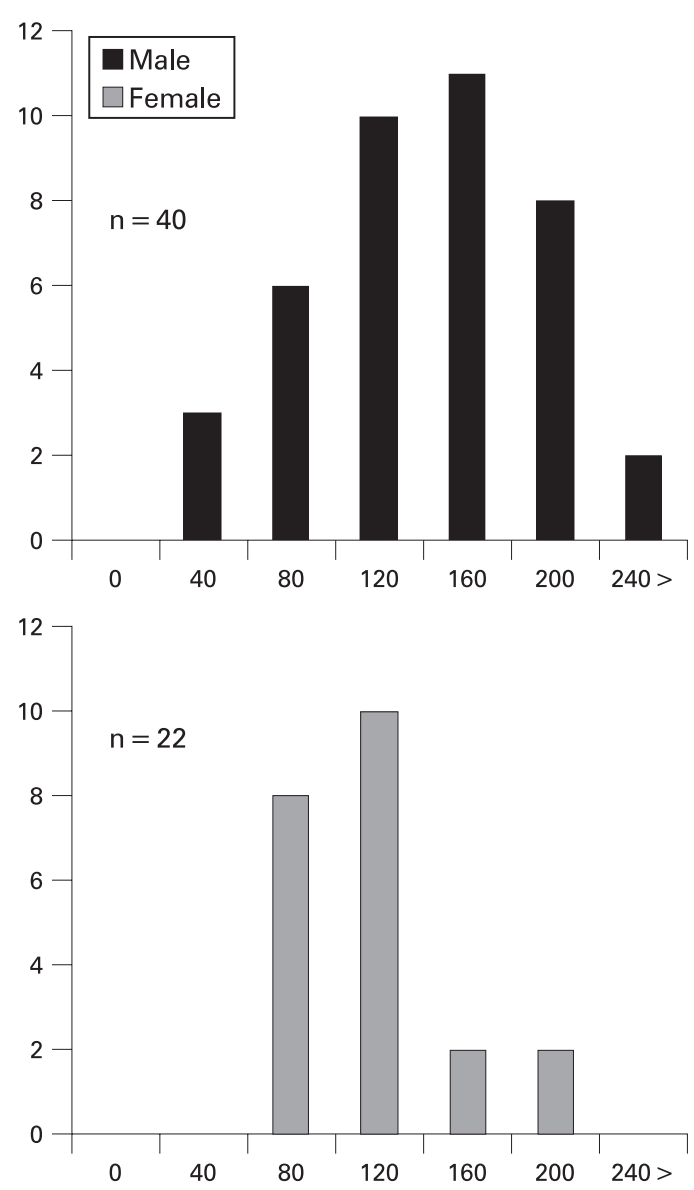

Figure 1 The mean value of the visceral fat area among male and female patients was 120.3 and $93.5 \mathrm{~cm}^{2}$, respectively.

platelet counts, HCC-specific biomarkers-that is, AFP, DCP and AFP-L3 - and liver function as classified by Child-Pugh scores (5-6 points, class A; 7-9 points, class B; and 10-12 points, class $\mathrm{C}$ ) based on albumin, total bilirubin, prothrombin activity, and the presence of ascites or hepatic encephalopathy. Nominal categorical data were represented by corresponding binary dummy variables. Categorical variables were compared by $\chi^{2}$ test (not ordered) or Cochran-Armitage test (ordered). Continuous variables were compared with unpaired Student $t$ test (parametric) or Wilcoxon test (non-parametric). Data processing and analysis were performed using the S-PLUS 2000 (MathSoft, Seattle, Washington, USA).

\section{RESULTS}

\section{Patient profile}

The patients were divided into two groups according to the cutoff levels of VFA recommended by Oka et al for metabolic syndrome in the Japanese population. ${ }^{18}$ The distribution of VFA in the present study, when shown separately in men and women, was similar to the previous data (fig 1). The mean VSA (SD) among male patients was $120.3(56.6) \mathrm{cm}^{2}$ and that among female patient was $93.5(37.8) \mathrm{cm}^{2}(p=0.036)$. The baseline characteristics of each group were shown in table 1 . The mean follow-up period was 3.4 (1.9) years in the high VFA group and 3.5 (1.9) years in controls $(p=0.95)$. The mean age was 67.5 (8.9) years in the high VFA group and 69.6 (9.7) years in controls $(p=0.37)$. The proportion of obese patients was significantly larger in the high VFA group $(p=0.001)$. However, there were no significant differences in the prevalence of comorbidities, diabetes mellitus and dyslipidaemia $(p=0.52$ and $p=0.78$, respectively). Hepatitis $\mathrm{B}$ core antibody ( $\mathrm{HBcAb}$ ) was positive in $8(29.6 \%)$ patients in the high VFA group and 10 patients $(28.6 \%)$ in the controls $(p=0.93)$. Other parameters were not significantly different between the two groups.

\section{Histology of the non-tumorous liver}

Histological diagnosis of NASH is often difficult at the time of HCC development due to decreased fat deposition in hepatocytes, especially when cirrhosis has been established. Histology of the non-tumorous liver was available in 60 of 62 patients $(26$ of the high VFA group and 34 of the controls), and we compared NAFLD activity score and fibrosis stages between the two groups (table 2). There were 6 (23.1\%) patients who were diagnosed as having NASH (NAFLD activity score $\geqslant 5$ ) among the high VFA group and $7(20.6 \%)$ patients among the controls $(p=0.92)$. Cirrhosis was identified in $18(69.2 \%)$ patients among the high VFA group and 20 (58.8\%) patients in the controls $(p=0.43)$.

\section{Percutaneous ablation}

Complete ablation was achieved in all patients, and major complications were identified in $3 / 27(11.1 \%)$ in the high VFA group and $1 / 35(2.9 \%)$ in the controls $(p=0.19)$ : neoplastic seeding $(n=1)$, liver infarction $(n=2)$ and liver abscess $(n=1)$. There was no treatment-related death. Although statistical consideration may not be appropriate because of the small size of the studied population, the safety and efficacy of RFA were considered to be acceptable in spite of obesity frequently found in the studied patients.

\section{Analysis of recurrence}

By the end of the follow-up, tumour recurrence was identified in $15 / 27(55.6 \%)$ patients in the high VFA group and in $11 / 35$ $(31.4 \%)$ in the controls. The cumulative recurrence rates at 1,2 and 3 years were $15.9,56.5$ and $75.1 \%$, respectively, in the high VFA group and 9.7, 31.1 and $43.1 \%$, respectively, in the controls (fig 2). The difference between the two groups was statistically significant $(p=0.018)$. Local tumour progression was found only in two patients in the high VFA group, who were treated as censored in the analysis of recurrence. The diameter of

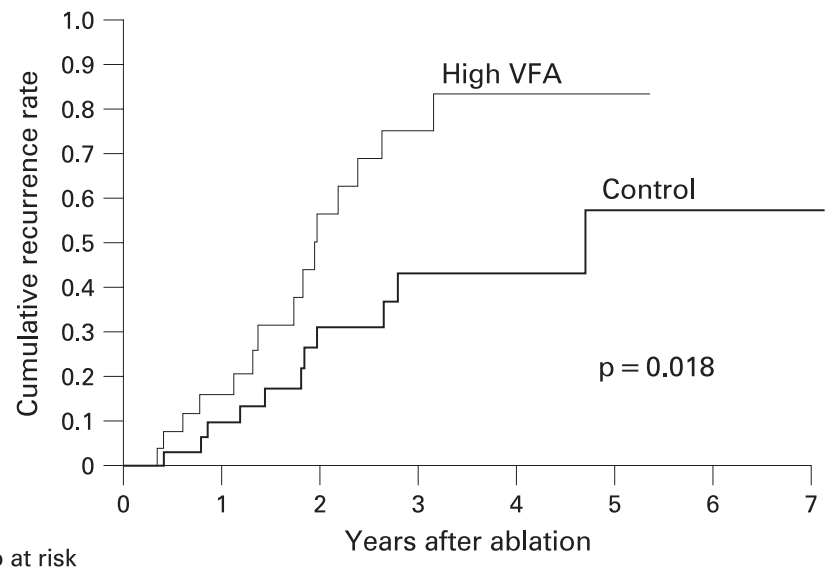

$\begin{array}{lllclll}\text { High VFA } & 27 & 19 & 7 & 3 & 1 & 1 \\ \text { Control } & 35 & 27 & 15 & 9 & 6 & 3\end{array}$

Figure 2 The cumulative recurrence rates at 1, 2 and 3 years were $15.9,56.5$ and $75.1 \%$, respectively, in the high visceral fat area (VFA) group and 9.7, 31.1 and $43.1 \%$, respectively, in the controls $(p=0.018)$. 
Table 1 Baseline characteristics of patients

\begin{tabular}{|c|c|c|c|}
\hline Variables (No) & High VFA group $(n=27)$ & Control group $(n=35)$ & p Value \\
\hline Age $^{*}$ & $67.5(8.9)$ & $69.6(9.7)$ & $0.37 \S$ \\
\hline Male & $16(59.3 \%)$ & $24(68.6 \%)$ & $0.59 \%$ \\
\hline Diabetes mellitus & $13(48.1 \%)$ & $14(40.0 \%)$ & $0.52 \ddagger$ \\
\hline Dyslipidaemia & $7(25.9 \%)$ & $8(22.9 \%)$ & $0.78 \%$ \\
\hline BMI $\left(\mathrm{kg} / \mathrm{m}^{2}\right)^{*}$ & $26.9(3.8)$ & $23.8(3.2)$ & $0.001 \S$ \\
\hline VFA $\left(\mathrm{cm}^{2}\right)^{*}$ & $152.1(45.2)$ & $79.0(29.9)$ & $0.001 \S$ \\
\hline SFA $\left(\mathrm{cm}^{2}\right)^{*}$ & $180.2(96.0)$ & $113.9(56.2)$ & $0.001 \S$ \\
\hline Waist circumference $(\mathrm{cm})^{*}$ & $92.5(10.0)$ & $84.2(10.6)$ & $0.001 \S$ \\
\hline Serum albumin $(\mathrm{g} / \mathrm{dl}) \dagger$ & $3.9(3.5-4.2)$ & $3.8(3.4-4.1)$ & $0.40 \S$ \\
\hline AST $(I U / I) \dagger$ & $39.0(27.5-50.0)$ & $38.0(24.5-53.5)$ & $0.94 \S$ \\
\hline Total bilirubin $(\mathrm{mg} / \mathrm{dl}) \dagger$ & $0.8(0.6-1.0)$ & $0.8(0.6-1.5)$ & $0.31 \S$ \\
\hline Platelet count $\left(\times 10^{3} / \mu l\right)$ & 135 (102-187) & 137 (122-189) & $0.96 \S$ \\
\hline Prothrombin activity $(\%) \dagger$ & $75.0(67.5-83.8)$ & $78.0(63.3-88.8)$ & $0.68 \S$ \\
\hline Child-Pugh classification & & & $0.30^{\oplus}$ \\
\hline Class A & $22(81.5 \%)$ & $26(74.3 \%)$ & \\
\hline Class B & $5(8.5 \%)$ & $6(17.1 \%)$ & \\
\hline Class C & $0(0 \%)$ & $3(8.6 \%)$ & \\
\hline HBcAb positive & $8(29.6 \%)$ & $10(28.6 \%)$ & $0.93 \%$ \\
\hline Tumour size $(\mathrm{mm})^{*}$ & $32.1(15.1)$ & $27.6(15.8)$ & $0.26 \S$ \\
\hline Number of nodules & & & $0.45 *$ \\
\hline UninodularUninodular & $16(59.3 \%)$ & $24(68.6 \%)$ & \\
\hline MultinodularMultinodular & $11(40.7 \%)$ & $11(31.4 \%)$ & \\
\hline AFP $>100 \mathrm{ng} / \mathrm{ml}$ & $4(14.8 \%)$ & $6(17.1 \%)$ & $0.80 \%$ \\
\hline $\mathrm{DCP}>100 \mathrm{mAU} / \mathrm{ml}$ & $5(18.5 \%)$ & $7(20.0 \%)$ & 0.88 \\
\hline AFP-L3 >15\% & $4(14.8 \%)$ & $6(17.1 \%)$ & $0.80 \%$ \\
\hline
\end{tabular}

\footnotetext{
${ }^{*}$ Expressed as mean (SD). †Expressed as median (25th-75th percentiles). $\$ \chi^{2}$ tests. §Unpaired Student $t$ test. $ๆ$ Cochran-Armitage test.

AFP, alpha-fetoprotein; AFP-L3, lens culinaris agglutinin-reactive fraction of AFP; AST, aspartate transaminase; BMI, body mass index; DCP, des-gamma-carboxy prothrombin; HBcAb, hepatitis B core antibody; SFA, subcutaneous fat area; VFA, visceral fat area.
}

recurrent tumour nodules was 18.9 (7.1) $\mathrm{mm}$ in the high VFA group and $23.7(10.8) \mathrm{mm}$ in the controls $(p=0.30)$, and the number of nodules was $1.4(0.8)$ in the high VFA group and 2.2 (1.8) in the controls $(P=0.42)$. By the end of follow-up, a total of 26 patients had HCC recurrence. The mean VFA at the first diagnosis of HCC and that at recurrence were 121.5 (52.0) and $129.5(79.8) \mathrm{cm}^{2}$, respectively ( $p=0.27$ by paired t test).

Univariate analysis identified older age $(p=0.006)$ and VFA $(p=0.022)$ as significant predictors for recurrence after curative ablation (table 3). BMI, SFA, waist circumference, comorbidity with diabetes mellitus, presence of dyslipidaemia and other variables did not show statistical significance at the $p<0.05$ level. Gender, although not significant in univariate analysis, was included in the multivariate analysis because the distribution of VFA was significantly different between men and women (fig 1). We also included comorbidity with diabetes mellitus and dyslipidaemia as variables to elucidate whether VFA was an independent risk factor for HCC recurrence. As a

Table 2 Histology of the non-tumorous liver

\begin{tabular}{llrl}
\hline Variables (No) & $\begin{array}{l}\text { High VFA group } \\
(\mathbf{n}=\mathbf{2 6})\end{array}$ & $\begin{array}{l}\text { Control group } \\
(\mathbf{n}=\mathbf{3 4})\end{array}$ & p Value \\
\hline NAFLD activity score & & & $0.92^{*}$ \\
$0-2$ & $17(65.4 \%)$ & $22(64.7 \%)$ & \\
$3-4$ & $3(11.5 \%)$ & $5(14.7 \%)$ & \\
$5-8$ (NASH) & $6(23.1 \%)$ & $7(20.6 \%)$ & \\
Cirrhosis & $18(69.2 \%)$ & $20(58.8 \%)$ & $0.43^{*}$
\end{tabular}

\footnotetext{
${ }^{*} \chi^{2}$ tests.

NAFLD, non-alcoholic fatty liver disease; NASH, non-alcoholic steatohepatitis;

VFA, visceral fat area.
}

result, only age and VFA retained significance in the multivariate analysis (table 4).

\section{Analysis of survival}

There were 14 deaths during the observation period: 6 deaths in the high VFA group and 8 deaths in the control group. The causes of death were HCC progression in 9, hepatic failure in 2, upper gastrointestinal bleeding in 1 and liver-unrelated causes in 2 patients. There was no significant difference in cumulative survival rate between the two groups $(p=0.99)$.

\section{DISCUSSION}

Currently in Japan, HCV infection is the most common background liver disease of HCC, followed by HBV infection and alcoholic liver disease. Primary biliary cirrhosis and other chronic liver diseases are an uncommon cause of HCC. The underlying liver disease in the remaining HCC cases, constituting about $5 \%$ of the total, is usually not determined, with histological examination revealing only cryptogenic cirrhosis. $\mathrm{NASH}$ is thought to be responsible for a substantial portion of such HCC cases, but characteristic pathology is rarely present at the stage of HCC development.

HCC is characterised by extremely frequent intrahepatic recurrence even after successful curative treatments. ${ }^{24}$ Both surgical hepatectomy and medical ablation are locoregional treatment in that the background liver diseases are left untreated. Liver transplantation forms an exception, after which recurrence is rare if the indication is appropriate. Two modes of intrahepatic recurrence have been distinguished: de novo carcinogenesis and intrahepatic metastasis. ${ }^{25}$ The factors responsible for the development of primary HCC, such as age, 
Table 3 Univariate analysis of recurrence after curative ablation

\begin{tabular}{lll}
\hline Variables & OR (95\% Cl) & p Value \\
\hline Age (per 1 year) & $1.06(1.02$ to 1.10$)$ & 0.006 \\
Male vs female & $0.81(0.37$ to 1.76$)$ & 0.60 \\
Diabetes mellitus & $1.49(0.69$ to 3.22$)$ & 0.30 \\
Dyslipidaemia & $2.01(0.69$ to 5.86$)$ & 0.20 \\
BMI (per $\left.1.0 \mathrm{~kg} / \mathrm{m}^{2}\right)$ & $1.04(0.92$ to 1.16$)$ & 0.53 \\
VFA (per $\left.10 \mathrm{~cm}^{2}\right)$ & $1.07(1.01$ to 1.14$)$ & 0.022 \\
SFA (per $\left.10 \mathrm{~cm}^{2}\right)$ & $1.02(0.97$ to 1.07$)$ & 0.40 \\
Waist circumference (per $1.0 \mathrm{~cm})$ & $1.01(0.98$ to 1.05$)$ & 0.56 \\
Serum albumin (per $1.0 \mathrm{~g} / \mathrm{dL})$ & $0.64(0.30$ to 1.38$)$ & 0.25 \\
AST (per $10 \mathrm{IU} /$ ) & $0.95(0.80$ to 1.13$)$ & 0.54 \\
Total bilirubin (per $1.0 \mathrm{mg} / \mathrm{dl})$ & $0.44(0.14$ to 1.37$)$ & 0.16 \\
Platelet counts (per $\left.10 \times 10^{3} / \mu l\right)$ & $0.96(0.90$ to 1.03$)$ & 0.25 \\
Prothrombin activity (per $1.0 \%)$ & $0.99(0.97$ to 1.02$)$ & 0.67 \\
HBcAb positive & $1.68(0.67$ to 4.18$)$ & 0.27 \\
Tumour size (per $1.0 \mathrm{~mm})$ & $1.00(0.98$ to 1.03$)$ & 0.84 \\
Multinodular vs. Uninodular & $1.57(0.70$ to 3.50$)$ & 0.27 \\
AFP $>100 \mathrm{ng} / \mathrm{ml}$ & $1.58(0.63$ to 4.02$)$ & 0.33 \\
DCP $>40 \mathrm{mAU} / \mathrm{ml}$ & $2.12(0.98$ to 4.62$)$ & 0.056 \\
AFP-L3 $>15 \%$ & $1.47(0.59$ to 3.68$)$ & 0.41 \\
\hline
\end{tabular}

AFP, alpha-fetoprotein; AFP-L3, lens culinaris agglutinin-reactive fraction of AFP; AST, aspartate aminotransferase; BMI, body mass index; DCP, des-gamma-carboxy prothrombin; $\mathrm{HBCAb}$, hepatitis $B$ core antibody; SFA, subcutaneous fat area; VFA, visceral fat area.

sex, fibrosis stage and the presence of viral hepatitis, will also affect de novo carcinogenesis, ${ }^{9}$ whereas the factors related to the primary HCC, such as the size and number of tumours, pathological grade and the presence of vascular invasion, will affect the possibility of intrahepatic metastasis. ${ }^{26}{ }^{27}$

In the present study, multivariate analysis showed older age and accumulation of visceral fat as independent risk factors for recurrence of non- $B$ non- $C$ non-alcoholic $H C C$ after curative ablation. The former factor was compatible with previous reports, whereas the relationship between visceral fat accumulation and the risk of HCC recurrence was a new finding in the present study. It may be suspected that visceral fat accumulation affected the efficacy of RFA. However, there were only two cases of local tumour progression, the most common sequel of insufficient ablation, which was not considered as an event but was censored in the present study. Thus, patients with high VFA showed a higher incidence of intrahepatic recurrence distant from the primary lesion, suggesting that visceral fat accumulation was associated with metachronous de novo carcinogenesis.

In the current study the risk of recurrence of non- $B$ non- $C$ HCC was associated with VFA but not with BMI, suggesting that non-B non-C hepatocarcinogenesis is more strongly associated with visceral fat accumulation than with BMI. In contrast to BMI, visceral fat accumulation is considered to be directly causative, through disturbing the adipocytokine balance of insulin resistance, which is a major cause of hepatic fat

Table 4 Multivariate analysis of recurrence after curative ablation

\begin{tabular}{lll}
\hline Variables & OR (95\% Cl) & p Value \\
\hline Age (per 1 year) & $1.07(1.03$ to 1.13$)$ & 0.003 \\
Diabetes mellitus & $2.04(0.89$ to 4.63$)$ & 0.093 \\
Dyslipidaemia & $1.99(0.66$ to 6.06$)$ & 0.225 \\
VFA (per $\left.10 \mathrm{~cm}^{2}\right)$ & $1.08(1.01$ to 1.17$)$ & 0.036 \\
Male vs female & $1.90(0.84$ to 4.33$)$ & 0.126 \\
\hline
\end{tabular}

VFA, visceral fat area. accumulation. ${ }^{28}$ Excessive fat in the liver will lead to hepatocellular injury and possibly result in hepatocarcinogenesis through the direct cellular toxicity of excessive free fatty acids, oxidant stress and lipid peroxidation, or another mechanism. ${ }^{10}$ Furthermore, visceral fat accumulation-induced insulin resistance causes hyperinsulinaemia. Insulin has growth-promoting properties and increases free insulin-like growth factor levels, which plays an important role in tumour growth and differentiation. ${ }^{29}{ }^{30}$ Visceral fat accumulation may be involved in both tumour initiation and promotion or progression steps through these mechanisms.

Since the present study examined recurrence of HCC, the results may not be applicable to primary hepatocarcinogenesis. In particular, there is the possibility that BMI and comorbidity with diabetes mellitus are significantly associated with the risk of HCC among patients with non-B non-C non-alcoholic liver diseases in general. Since the presence of chronic hepatic inflammation is a prerequisite for hepatocarcinogenesis, BMI and comorbidity with diabetes mellitus, which are associated with the risk of NASH, could still be used as an indicator of the risk of HCC development among patients with NAFLD. ${ }^{10}$ On the other hand, the subjects of the present study, who had developed HCC, had a definite risk of de novo carcinogenesis. BMI and comorbidity with diabetes mellitus are not a significant predictor among such patients.

The distribution of visceral fat is different between males and females, ${ }^{31}$ and the present study showed similar data: average VFA was different between men (120.3 (56.6) $\left.\mathrm{cm}^{2}\right)$ and women (93.5 (37.8) $\mathrm{cm}^{2}, \mathrm{p}=0.036$ ). According to this result, we defined the high VFA group as VFA $>130 \mathrm{~cm}^{2}$ in males and VFA $>90 \mathrm{~cm}^{2}$ in females. These values are equal to the cut-offs recommended by Oka et al for metabolic syndrome in the Japanese population. ${ }^{18}$ Thus, it can be speculated that mechanisms involved in metabolic syndrome played a greater role in the hepatocarcinogenesis among patients with high VFA than in that among the others.

In conclusion, visceral fat accumulation was shown to be an independent risk factor of recurrence in patients with non-B non-C non-alcoholic HCC treated with curative ablation. Visceral fat accumulation plays a significant role in de novo hepatocarcinogenesis among such patients, and it remains to be seen whether reduction of visceral fat decreases HCC recurrence.

Competing interests: None.

\section{REFERENCES}

1. El-Serag HB, Mason AC. Rising incidence of hepatocellular carcinoma in the United States. N Engl J Med 1999;340:745-50.

2. Bosch FX, Ribes J, Diaz M, et al. Primary liver cancer: worldwide incidence and trends. Gastroenterology 2004;127:S5-16.

3. Shiratori Y, Shiina S, Imamura M, et al. Characteristic difference of hepatocellular carcinoma between hepatitis B- and C- viral infection in Japan. Hepatology 1995:22:1027-33.

4. El-Serag HB. Hepatocellular carcinoma: recent trends in the United States. Gastroenterology 2004;127:S27-34.

5. Charlton M. Nonalcoholic fatty liver disease: a review of current understanding and future impact. Clin Gastroenterol Hepatol 2004;2:1048-58.

6. Ludwig J, Viggiano TR, McGill DB, et al. Nonalcoholic steatohepatitis: Mayo Clinic experiences with a hitherto unnamed disease. Mayo Clin Proc 1980;55:434-8.

7. Ioannou GN, Splan MF, Weiss NS, et al. Incidence and predictors of hepatocellular carcinoma in patients with cirrhosis. Clin Gastroenterol Hepatol 2007;5:938-45.

8. Nair S, Mason A, Eason J, et al. Is obesity an independent risk factor for hepatocellular carcinoma in cirrhosis? Hepatology 2002;36:150-5.

9. Ohki T, Tateishi R, Sato T, et al. Obesity is an independent risk factor for hepatocellular carcinoma development in chronic hepatitis $\mathrm{C}$ patients. Clin Gastroenterol Hepatol 2008;6:459-64.

10. Neuschwander-Tetri BA, Caldwell SH. Nonalcoholic steatohepatitis: summary of an AASLD Single Topic Conference. Hepatology 2003;37:1202-19. 
11. Eguchi Y, Eguchi T, Mizuta T, et al. Visceral fat accumulation and insulin resistance are important factors in nonalcoholic fatty liver disease. J Gastroenterol 2006;41:462-9.

12. Teratani T, Yoshida H, Shiina S, et al. A novel display of reconstruction computed tomography for the detection of small hepatocellular carcinoma. Liver Int 2004;24:619-24.

13. Fujishima T, Yoshida $\mathrm{H}, \mathrm{Obi} \mathrm{S}$, et al. Analysis of factors influencing hepatocellular carcinoma detection: efficient use of computed tomography during arterial portography and during hepatic arteriography. J Gastroenterol 2005;40:266-73.

14. Nonalcoholic steatohepatitis clinical research network. Hepatology 2003;37:244.

15. Ishak K, Baptista A, Bianchi $L$, et al. Histological grading and staging of chronic hepatitis. J Hepatol 1995;22:696-9.

16. Edmondson HA, Steiner PE. Primary carcinoma of the liver: a study of 100 cases among 4900 necropsies. Cancer 1954:7:462-503.

17. Zhao B, Colville J, Kalaigian J, et al. Automated quantification of body fat distribution on volumetric computed tomography. J Comput Assist Tomogr 2006;30:777-83

18. Oka R, Kobayashi J, Yagi K, et al. Reassessment of the cutoff values of waist circumference and visceral fat area for identifying Japanese subjects at risk for the metabolic syndrome. Diabetes Res Clin Pract 2008;79:474-81.

19. Omata M, Tateishi R, Yoshida H, et al. Treatment of hepatocellular carcinoma by percutaneous tumor ablation methods: ethanol injection therapy and radiofrequency ablation. Gastroenterology 2004;127:S159-66.

20. Jaskolka JD, Asch MR, Kachura JR, et al. Needle tract seeding after radiofrequency ablation of hepatic tumors. J Vasc Interv Radiol 2005;16:485-91.

21. Llovet JM, Vilana R, Bru C, et al. Increased risk of tumor seeding after percutaneous radiofrequency ablation for single hepatocellular carcinoma. Hepatology 2001;33:1124-9
22. Mulier S, Mulier $\mathrm{P}, \mathrm{Ni} \mathrm{Y}$, et al. Complications of radiofrequency coagulation of liver tumours. Br J Surg 2002;89:1206-22.

23. Fine JP, Gray RJ. A proportional hazards model for the subdistribution of a competing risk. J Am Stat Assoc 1999:94:496-506.

24. Tateishi R, Shiina S, Yoshida H, et al. Prediction of recurrence of hepatocellular carcinoma after curative ablation using three tumor markers. Hepatology 2006;44:1518-27.

25. Sakon M, Umeshita $\mathrm{K}$, Nagano $\mathrm{H}$, et al. Clinical significance of hepatic resection in hepatocellular carcinoma: analysis by disease-free survival curves. Arch Surg 2000; 135:1456-9.

26. Koike Y, Shiratori $Y$, Sato $S$, et al. Risk factors for recurring hepatocellular carcinoma differ according to infected hepatitis virus - an analysis of 236 consecutive patients with a single lesion. Hepatology 2000;32:1216-23.

27. Ohki T, Tateishi R, Shiina $S$, et al. Obesity did not diminish the efficacy of percutaneous ablation for hepatocellular carcinoma. Liver Int 2007;27:360-7.

28. Lo lacono $\mathbf{0}$, Venezia $\mathrm{G}$, Petta $\mathrm{S}$, et al. The impact of insulin resistance, serum adipocytokines and visceral obesity on steatosis and fibrosis in patients with chronic hepatitis C. Aliment Pharmacol Ther 2007;25:1181-91.

29. Giovannucci E, Michaud D. The role of obesity and related metabolic disturbances in cancers of the colon, prostate, and pancreas. Gastroenterology 2007;132:2208-25

30. Alexia C, Fallot $\mathrm{G}$, Lasfer $\mathrm{M}$, et al. An evaluation of the role of insulin-like growth factors (IGF) and of type-I IGF receptor signalling in hepatocarcinogenesis and in the resistance of hepatocarcinoma cells against drug-induced apoptosis. Biochem Pharmacol 2004;68:1003-15.

31. Fox CS, Massaro JM, Hoffmann U, et al. Abdominal visceral and subcutaneous adipose tissue compartments: association with metabolic risk factors in the Framingham Heart Study. Circulation 2007;116:39-48. 